\title{
Study of J-E Curve with Hysteresis of Carbon Nanotubes Field Emitters
}

\author{
Shama Parveen, Samina Husain, Avshish Kumar, Javid Ali, Harsh, and Mushahid Husain \\ Department of Physics, Jamia Millia Islamia, New Delhi 110025, India \\ Correspondence should be addressed to Mushahid Husain, mush_reslab@rediffmail.com \\ Received 7 June 2012; Accepted 15 July 2012 \\ Academic Editors: J. Escrig, G. Jin, and A. Kajbafvala \\ Copyright () 2012 Shama Parveen et al. This is an open access article distributed under the Creative Commons Attribution License, \\ which permits unrestricted use, distribution, and reproduction in any medium, provided the original work is properly cited.
}

\begin{abstract}
We observe hysteresis in J-E plot during field emission measurement of CNTs grown by LPCVD technique. CNTs are synthesized on Fe-coated Si substrate at $650^{\circ} \mathrm{C}$. SEM and Raman study confirm that CNTs are successfully grown on Si substrate by LPCVD technique. In this study, we find that ramp-down curve has higher value of current density than ramp-up curve which indicates that CNTs show positive hysteresis. Our results show that a high current density at low turn-on voltage is obtained in ramp-down step of J-E plot which may be since not all CNTs contribute in ramp-up step process. But in ramp-down step all CNTs contribute as field emitters due to high electric field treatment. We also performed stability analysis of CNTs with current at constant applied voltage for $5 \mathrm{hrs}$ and find that the sample shows long-term stability due to increase in emitting site density since a large number of CNTs participate in field emission.
\end{abstract}

\section{Introduction}

Carbon nanotube (CNT) is a very attractive material for researchers due to its fortunate combination of miniature size, high mechanical strength, good electrical and thermal conductivity, and also high thermal stability $[1,2]$. Moreover, an important feature of CNTs is their high aspect ratio. Due to this peculiarity, the applied electric field strength in the vicinity of the tip of the CNTs can exceed by thousand times [3]. This property, in turn, manifests CNTs in the possibility of obtaining a quite high current density at a moderate magnitude of applied voltage. A high field enhancement factor $(\beta)$ allows electrons to overcome the surface potential barrier at low macroscopic electric fields. Hence CNT-based electron field emitter cathodes are superior to other types of electron field emitter in their emission characteristics [4].

These excellent field emission (FE) characteristics have made CNT emitters the center of researcher's attention for various potential applications. CNTs as electron field emitter source in various fields have been widely studied such as cold cathodes for field emission displays (FEDs) [5], scanning probe for atomic force microscope (AFM) [6], electron source for vacuum microelectronics (VME), X-ray source for computed tomography (CT) scanners. CNT-based vacuum microelectronic and display devices have also been already proposed [7-14].

In present work, we study the hysteresis loop during FE measurements to enhance the FE characteristics of CNT cathode. It provides us very effective results in order to get the high current density at low turn-on field $\left(E_{\mathrm{TO}}\right)$, and at the same time it does not require any external special arrangement to remove the amorphous carbon on the CNTs surface. Here, CNTs are synthesized by low-pressure chemical vapor deposition (LPCVD) technique which is one of the best techniques for the growth of CNTs with fewer impurities. Iron ( $\mathrm{Fe}$ ) is used as catalyst and helps to make a good adhesion between CNT and the substrate. FE measurements are taken in ramp-up and ramp-down steps. The measurements show positive hysteresis characteristics of as-grown CNTs which implies that FE characteristics have improved due to positive hysteresis. From JE curve, we also find that $E_{\mathrm{TO}}$ decreases with high current density. Furthermore, on application of constant applied voltage, CNTs remain stable as they continue to emit electrons for 5 hrs. Repeatability measurements of the sample show that FE characteristic of CNTs enhances after ramp-down step process. 


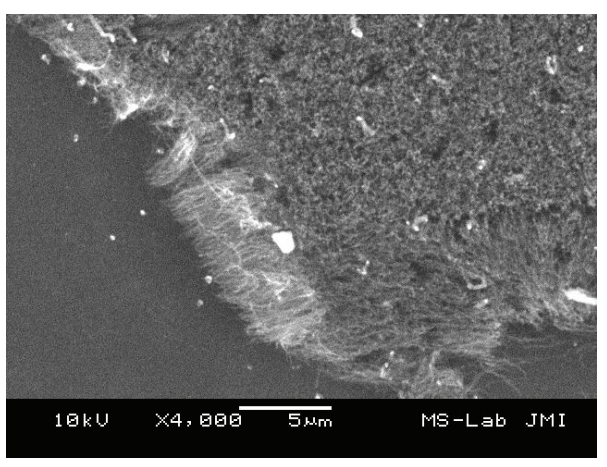

(a)

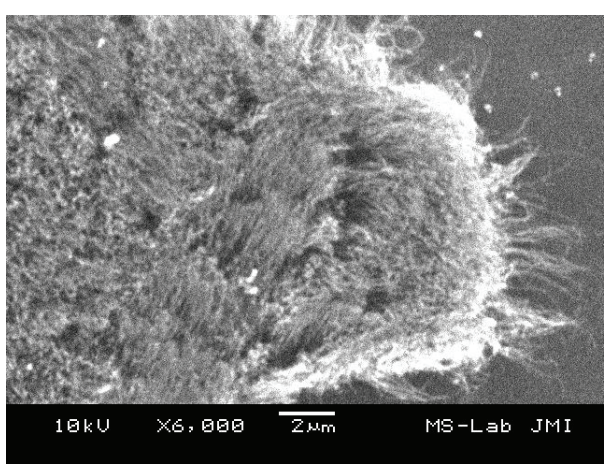

(b)

FIGURE 1: (a) SEM image of CNTs grown by LPCVD, (b) SEM image with enlarged view of CNTs grown by LPCVD.

\section{Experimental}

CNTs have been synthesized by LPCVD technique on $\mathrm{Si}$ substrate. Fe catalyst is deposited on ultrasonically cleaned $\mathrm{Si}$ substrate by radio-frequency (RF) sputtering (MODEL: 12" MSPT) at a power of $150 \mathrm{~W}$. For synthesis of CNTs, quartz tube of LPCVD is cleaned first by purging nitrogen $\left(\mathrm{N}_{2}\right)$ gas. Catalyst-deposited Si substrate is placed in a $150 \mathrm{~cm}$ large quartz tube furnace at room temperature and is pumped down to $10^{-3}$ torr using rotary pump. Rough vacuum is necessary for low pressure growth and also to improve the purity of CNTs during growth process. The Fe catalyst-coated Si substrate is heated to achieve synthesis temperature at a rate of $15^{\circ} \mathrm{C} / \mathrm{min}$ while continuously passing the hydrogen $\left(\mathrm{H}_{2}\right)$ gas which works as carrier gas. After stabilizing the synthesis temperature at $650^{\circ} \mathrm{C}$, ammonia $\left(\mathrm{NH}_{3}\right)$ gas is passed with flow rate $120 \mathrm{sccm}$ for pretreatment which help in nucleation of Fe. Pretreatment reduces the size of catalyst particle up to nanometer level which provides nucleation site for formation of CNTs. A mixture of gases $\mathrm{H}_{2} / \mathrm{C}_{2} \mathrm{H}_{2} / \mathrm{NH}_{3}$ with flow rate $6: 1: 6 \mathrm{sccm}$, respectively, is used, where $\mathrm{C}_{2} \mathrm{H}_{2}$ works as a carbon source gas. Growth temperature is kept constant at $650^{\circ} \mathrm{C}$. The as-grown CNT film is characterized by Raman spectroscopy and scanning electron microscope (SEM). FE measurements are carried out using diode configuration with CNTs as cathode. Current density versus electric field (JE) and Fowler Nordheim (FN) plots associated with hysteresis loop is studied. Repeatability and stability are also performed in the same FE chamber. Field enhancement factor is also calculated from FN plot.

\section{Result and Discussion}

3.1. SEM Morphology. Figure 1(a) depicts surface morphology of as-grown CNTs on Fe catalyst at temperature $650^{\circ} \mathrm{C}$. The thickness of the catalyst layer on Si substrate is in the range of $10-25 \mathrm{~nm}$. It is evident from these SEM images that vertically aligned and high densities of CNTs have been successfully grown by LPCVD technique. Both are fundamental requirements for good field emission characteristics of CNTs. The growth morphology of nanotubes depends upon the size, shape, and surface nature of catalyst. The
CNTs obtained by this technique also have high aspect ratio with diameter varying from 10 to $30 \mathrm{~nm}$ and length of several micrometers. Figure $1(\mathrm{~b})$ is the enlarged view of Figure 1(a). SEM images also reveal that there are no bright spots of catalyst on the tip of as-grown CNTs film; therefore, base-growth mechanism is proposed here. In base-growth method, CNTs are nucleated from Fe catalyst particles in which catalyst particles remain adherent to the Si substrates during growth time. Because iron silicide roots penetrate into the Si substrate at the interface of the catalyst particles and the substrate, it favors strong adhesion of the CNTs onto the substrate. The iron silicide root assists in the attachment of the catalyst particles to the substrate and plays a role in the evolution of the catalyst particle morphology resulting in base-growth mode [7]. Base-growth of CNTs is also favored for high field emission property of CNTs because impurities like metal catalyst are not present on the tip of CNT field emitters.

3.2. Raman Spectroscopy Analysis. To investigate the graphitic structure of CNTs, the high-resolution spectrum in the high-frequency region shows two intensive peaks around $1350 \mathrm{~cm}^{-1}$ and $1580 \mathrm{~cm}^{-1}$. Generally, G-mode (TM-tangential mode) in Raman spectra corresponding to the stretching mode in graphitic plane is located around $1580 \mathrm{~cm}^{-1}$. The D mode (disorder band), located between 1330 and $1360 \mathrm{~cm}^{-1}$ is generally observed in MWCNTs. Figure 2 shows a typical Raman spectrum obtained by Raman spectrometer (Bruker, RFS 100/s) using Nd:YAG laser with an excitation wavelength of $1064 \mathrm{~nm}$. The spectra depict strong peak at $1590 \mathrm{~cm}^{-1}$ ( $\mathrm{G}$ band), suggesting the formation of graphitized CNTs which is common to all $\mathrm{sp}^{2}$ system. The peak observed at $1355 \mathrm{~cm}^{-1}$ (D band) indicates that there is some amorphous carbonaceous and some catalyst particles adhered to the CNTs walls and also few defective pentagons and heptagons exists in the graphitized walls due to low growth temperature. We also observe one additional peak at $2700 \mathrm{~cm}^{-1}$ which is attributed to second-order Raman scattering process. Intensity ratio of defective band and graphite band is a signature of quality of CNTs. As seen from Figure $2, I_{\mathrm{D}} / I_{\mathrm{G}}$ of as-grown CNTs is 0.852 which indicates that our sample is less defective. 


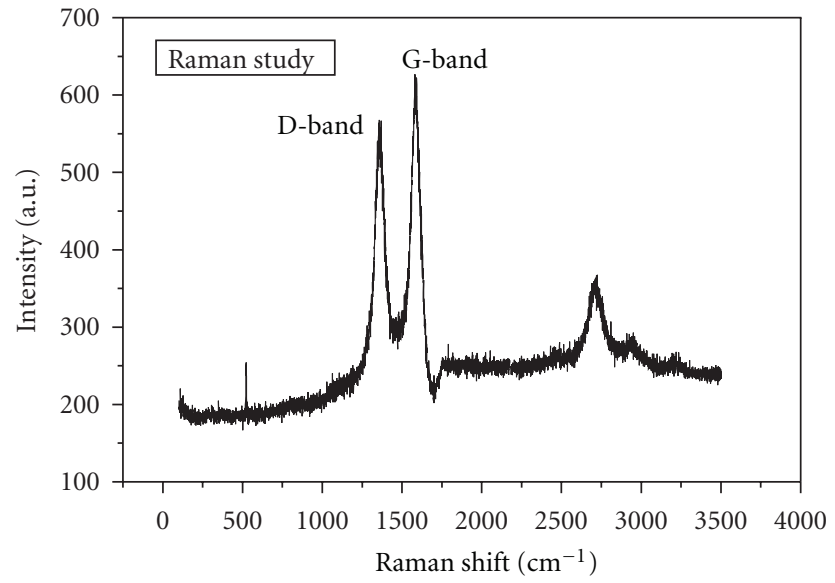

FIgURE 2: Raman Spectra of as-grown CNTs.

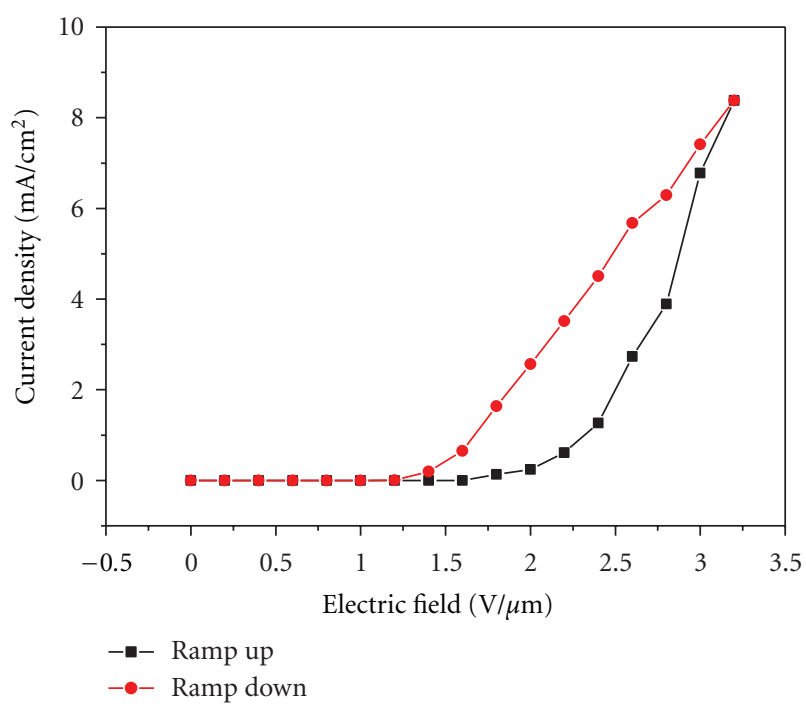

Figure 3: Current density versus electric field curve of ramp-up and ramp-down step.

3.3. Field Emission Analysis. Field emission measurements are usually analysed in the framework of the FowlerNordheim theory [12], which explains the emission of the electrons from emitter surface as a quantum mechanical tunnelling enhanced by a high electric field. The FN equation can be written as

$$
J=A E^{2} \exp \left(-\frac{B \phi^{3 / 2}}{E}\right),
$$

where $A=1.54 \times 10^{-6} \mathrm{Aev} \mathrm{V}^{-2}, B=6.83 \times 10^{7} \mathrm{ev}^{3 / 2} \mathrm{~V} \mathrm{~cm}^{-1}$, $E_{h}$ : electric field at the apex, $\mathrm{V} \mathrm{cm}^{-1}$, and $\phi$ : work function.

The enhanced electric field at the CNTs tip $E_{h}$ is related to the applied voltage $V$ via $E_{h}=\beta E_{\text {applied }}=\beta V / d, \beta$ being the field enhancement factor at the sharp tip of the CNTs and $d$ is the CNT cathode and anode separation.

Typical FE measurements for as-grown CNTs are depicted in Figure 3. Electric field versus current density, that is, JE plot, is shown in Figure 3. In present work, we study the JE plot in two-step process: one is ramp up

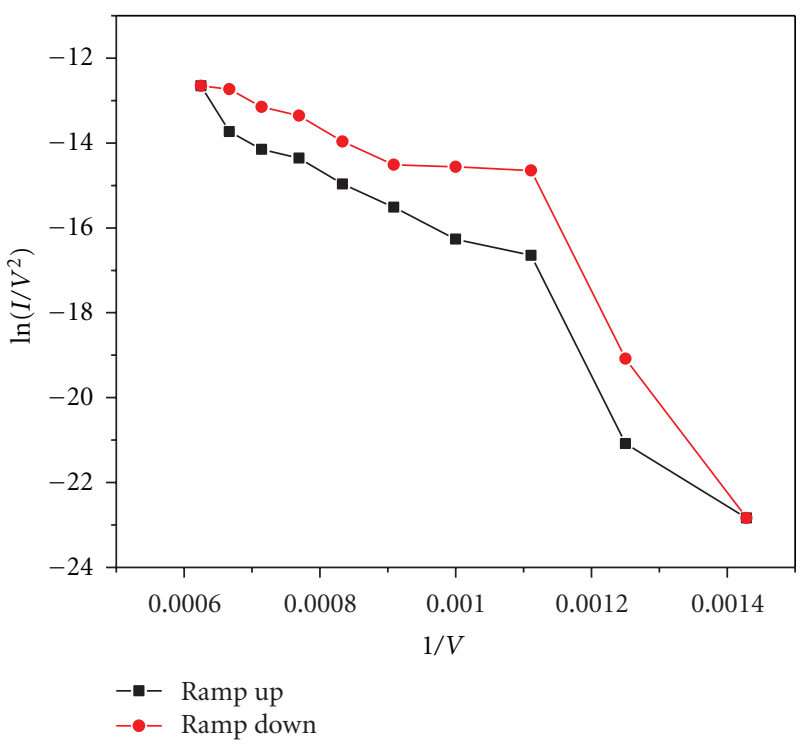

FIGURE 4: F-N plot with ramp-up and ramp-down step.

and other is ramp down. In ramp-up step, we note down the current density corresponding to increasing applied voltage from $100 \mathrm{~V}$ to $1500 \mathrm{~V}$. While in ramp-down step, the current density is taken corresponding to decreasing applied voltage from $1500 \mathrm{~V}$ to $100 \mathrm{~V}$. This ramp-up and rampdown step forms a loop which is known as hysteresis loop. Emission current density which is the function of applied macroscopic electric field reaches its maximum value at $8.1 \mathrm{~mA} / \mathrm{cm}^{2}$ and $E_{\mathrm{TO}}$ is achieved at $2.2 \mathrm{~V} / \mu \mathrm{m}$. However, $E_{\mathrm{TO}}$ field is obtained at $1.7 \mathrm{~V} / \mu \mathrm{m}$ in ramp-down step. It is worth noting that $E_{\mathrm{TO}}$ decreases in ramp-down step and current density corresponding to applied voltage as compared to ramp-up step increases. These results confirm that CNT cathode has the same hysteresis effect and also suggest that this hysteresis is associated with amorphous carbon and impurities presented on the surface of CNTs cathode [15]. In hysteresis loop, current density improvement may be due to application of applied high electric field. In ramp-up step, increasing voltage at the diode assembly removes amorphous carbon and other impurities that are present on the tip of CNTs.

Figure 4 depicts the FN plots of as-grown CNT cathodes. Almost straight line of FN plot confirms that field emission phenomenon is taking place in our sample. FN plots also show hysteresis loop. According to the FN model, a plot of $\ln \left(I / V^{2}\right)$ versus $1 / V$ (known as F-N plot) has a linear behavior with a slope that is also used to calculate $\beta$ by using the following simplified equation derived from FN:

$$
\beta=\frac{B \phi^{3 / 2} d}{m}
$$

where $m$ : slope of FN plot, $d$ : distance between cathode and anode, and $\phi=5 \mathrm{eV}$ as for carbon. Calculated values of $\beta$ are 3000 and 12500 for ramp up and ramp down, respectively. The high $\beta(\sim 4.2)$ value may also have resulted in improving the current density in hysteresis loop. So, this high-field treatment cleans the surface of the CNTs. This may also be 


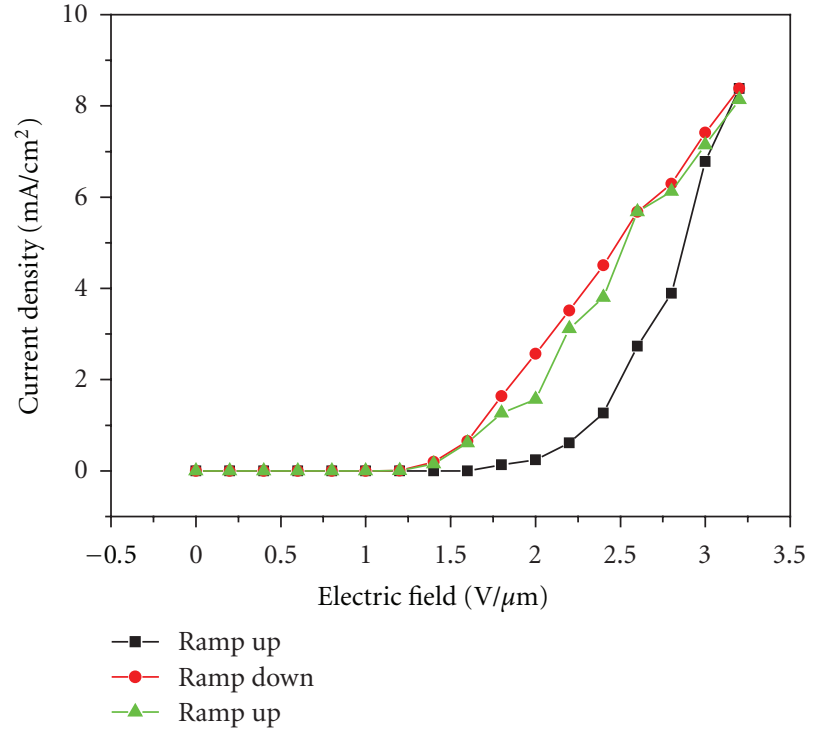

(a)

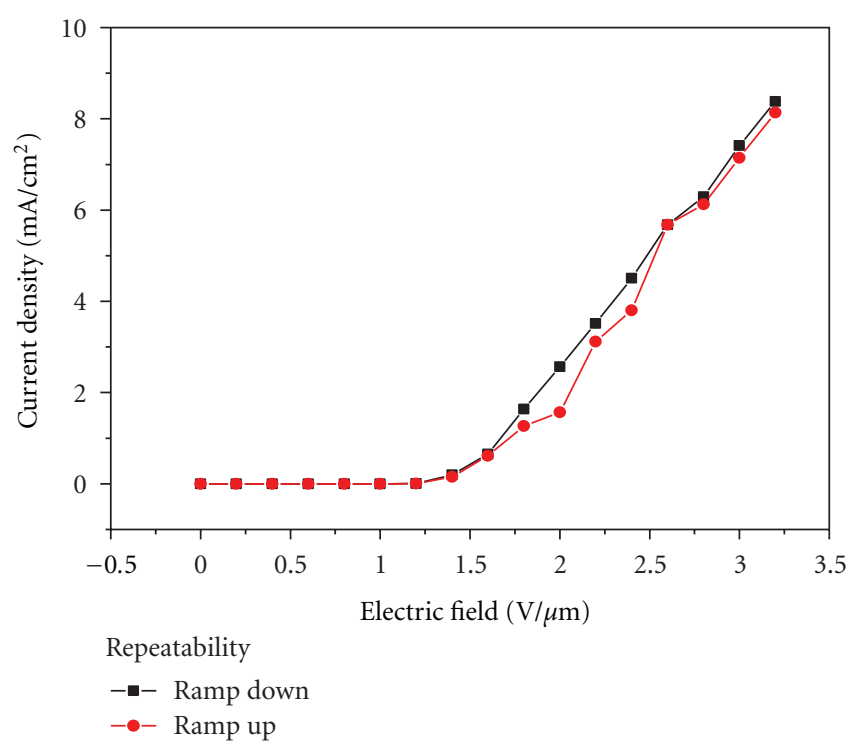

(b)

Figure 5: (a), (b): Repeatability of as-grown CNTs of J-E curve.

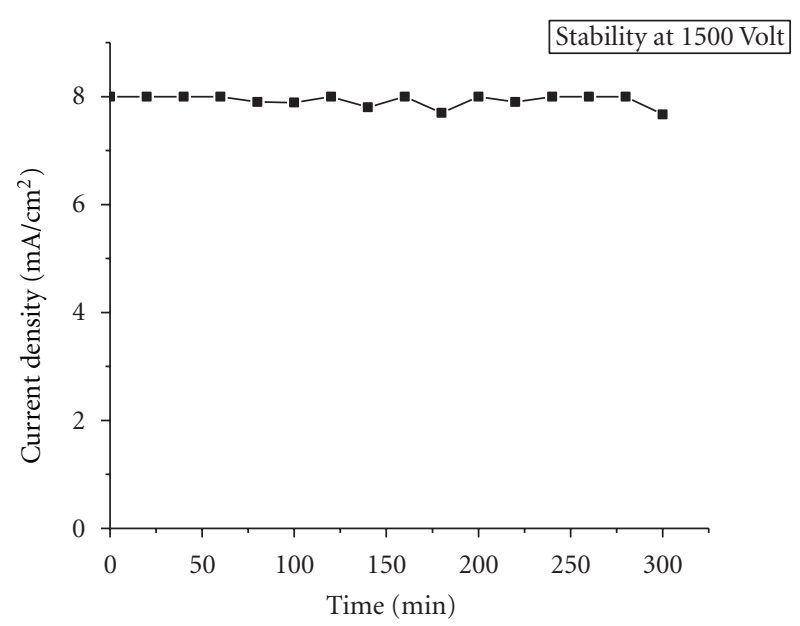

FIGURE 6: Emission current stability of CNTs operated in a dc mode at electric field $3 \mathrm{~V} / \mathrm{m}$ over $5 \mathrm{hrs}$.

attributed to a reason that amorphous carbon and other impurities might be removed from the surface of CNTs film on applying high electric field. This enhanced field hence, breaks molecules of residual gases into ions and exposes large area of CNTs film contributing to a large $\beta$. Thus it seems that in ramp-down process, large emitting area is available for emitting electrons than in ramp-up process, as a consequence of which current density enhances at low $E_{\mathrm{TO}}$.

3.4. Repeatability. We repeated the field emission measurement of JE plot several times and found a good match of ramp-up and ramp-down step. Therefore, this confirms that the emission characteristics show good repeatability shown in Figures 5(a) and 5(b).
3.5. Emission Stability. We evaluated the emission current stability of these CNT field emitters. The stability of emission was performed by monitoring the evolution of total emission current at a fixed electric field. The initial emission current density was about $8.1 \mathrm{~mA} \mathrm{~cm}^{-2}$ and the applied voltage was kept constant at $3 \mathrm{~V} / \mu \mathrm{m}$ for $5 \mathrm{hrs}$. A current density of $8.1 \mathrm{~mA} \mathrm{~cm}^{-2}$ is a severe condition to clearly visualize current degradation within a relatively short time. Figure 6 shows a more stable emission current without any noticeable degradation of the emission current density till over $5 \mathrm{hrs}$. We also confirmed this trend with good repeatability a number of times. The result shows that the greatly enhanced field emission stability is mainly caused by increase of the clean surface of emission sites due to the removal of the absorbents and residual gases on the surface of CNT film [16].

\section{Conclusions}

Repeatability of CNT-based field emission devices has been studied by hysteresis loop of JE plot. It is noteworthy that the suggested method enhances the field emission characteristics of CNT field emitters such as the $\beta, E_{\mathrm{TO}}$, and $E_{\mathrm{Th}}$, and even it also improves emission repeatability and stability. Thus the study of hysteresis loop is demonstrated to be an effective way to improve the FE characteristics of CNTs field emitters. It is considered that $E_{\mathrm{TO}}$ at $1.96 \mathrm{~V} / \mu \mathrm{m}$ is quite good and is sufficient for real applications. In addition, the electric field of $2.85 \mathrm{~V} / \mu \mathrm{m}$ for obtaining maximum emission current density of $8.1 \mathrm{~mA} \mathrm{~cm} \mathrm{~cm}^{-2}$ is high enough for device applications. Therefore, some sacrifice in both the stability and repeatability does not make critical problems for a real operation mode of CNTs field emitters. Thus, our results not only suggest the synthesis of CNTs at low temperature but also offer to enhance field emission parameter of CNT-based 
field emitters for various potential applications in nanoscale electronic devices.

\section{Acknowledgments}

Thanks are due to DIT, Government of India, for providing financial assistance in form of research project. One of the authors (S. Husain) is also thankful to CSIR, India, for providing financial support as research associateship.

\section{References}

[1] A. V. Eletskii, "Carbon nanotube-based electron field emitters," Physics-Uspekhi, vol. 53, no. 9, pp. 863-892, 2010.

[2] R. P. Raffaelle, B. J. Landi, J. D. Harris, S. G. Bailey, and A. F. Hepp, "Carbon nanotubes for power applications," Materials Science and Engineering B, vol. 116, no. 3, pp. 233-243, 2005.

[3] M. J. Fransen, T. L. van Rooy, and P. Kruit, "Field emission energy distributions from individual multiwalled carbon nanotubes," Applied Surface Science, vol. 146, no. 1, pp. 312 327, 1999.

[4] Y. Cheng and O. Zhou, "Electron field emission from carbon nanotubes," Comptes Rendus Physique, vol. 4, no. 9, pp. 10211033, 2003.

[5] J. M. Bonard, M. Croci, C. Klinke, R. Kurt, O. Noury, and N. Weiss, "Carbon nanotube films as electron field emitters," Carbon, vol. 40, no. 10, pp. 1715-1728, 2002.

[6] J. M. Bonard, F. Maier, T. Stöckli et al., "Field emission properties of multiwalled carbon nanotubes," Ultramicroscopy, vol. 73, no. 1-4, pp. 7-15, 1998.

[7] J. F. AuBuchon, C. Daraio, L. H. Chen, A. I. Gapin, and S. Jin, "Iron suicide root formation in carbon nanotubes grown by microwave PECVD," Journal of Physical Chemistry B, vol. 109, no. 51, pp. 24215-24219, 2005.

[8] S. Khan, K. N. Tripathi, M. Aggarwal, K. Tripathi, M. Husain, and $\mathrm{Z}$. H. Khan, "Field emission properties of $\mathrm{Fe}_{70} \mathrm{Pt}_{30}$ catalysed multiwalled carbon nanotubes," Journal of Experimental Nanoscience, vol. 2, no. 3, pp. 215-228, 2007.

[9] J. Ali, A. Kumar, S. Husain, and M. Husain, "Effect of catalystdeposition methods on the alignment of carbon nanotubes grown by low pressure chemical vapor deposition," Nanoscience and Nanotechnology Letters, vol. 3, no. 2, pp. 175-178, 2011.

[10] J. M. Rosolen, C. H. Patrick Poá, S. Tronto, M. S. Marchesin, and S. R. P. Silva, "Electron field emission of carbon nanotubes on carbon felt," Chemical Physics Letters, vol. 424, no. 1-3, pp. 151-155, 2006.

[11] S. Khan, Z. H. Khane, K. N. Tripathi, and M. Husain, "Synthesis of carbon nanotubes using $\mathrm{Ni}_{95} \mathrm{Ti}_{5}$ nanocrystalline film as a catalyst," Journal of Nanoscience and Nanotechnology, vol. 7, no. 6, pp. 1855-1859, 2007.

[12] S. Parveen, S. Husain, A. Kumar, J. Ali, and M. Husain, "Estimation of effective emitting area of carbon nanotubes based field emitters," Nanoscience and Nanotechnology Letters, vol. 3, pp. 794-797, 2011.

[13] J. Ali, A. Kumar, S. Husain, M. Kumari, Harsh, and M. Husain, "Characterization and field emission studies of uniformly distributed multi-walled carbon nanotubes (MWCNTS) film grown by low-pressure chemical vapour deposition (LPCVD)," Current Nanoscience, vol. 7, no. 3, pp. 333-336, 2011.
[14] Y. H. Yang, C. Y. Wang, U. S. Chen, W. J. Hsieh, Y. S. Chang, and H. C. Shih, "Large-area single wall carbon nanotubes: synthesis, characterization, and electron field emission," Journal of Physical Chemistry C, vol. 111, no. 4, pp. 1601-1604, 2007.

[15] C. Li, G. Fang, X. Yang, N. Liu, Y. Liu, and X. Zhao, "Effect of adsorbates on field emission from flame-synthesized carbon nanotubes," Journal of Physics D, vol. 41, no. 19, Article ID 195401, 2008.

[16] K. A. Dean and B. R. Chalamala, "The environmental stability of field emission from single-walled carbon nanotubes," Applied Physics Letters, vol. 75, no. 19, pp. 3017-3019, 1999. 

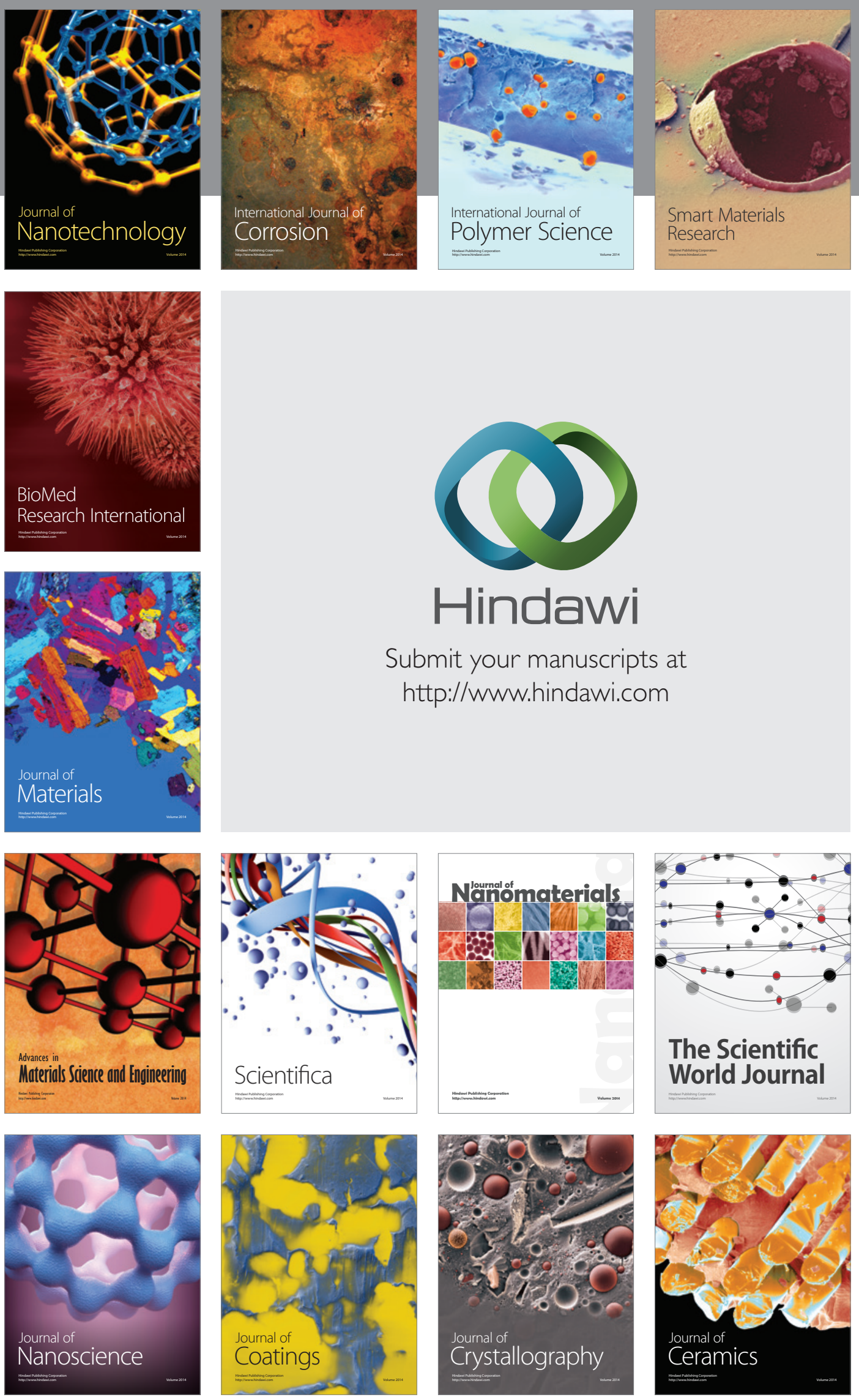

The Scientific World Journal

Submit your manuscripts at

http://www.hindawi.com

\section{World Journal}

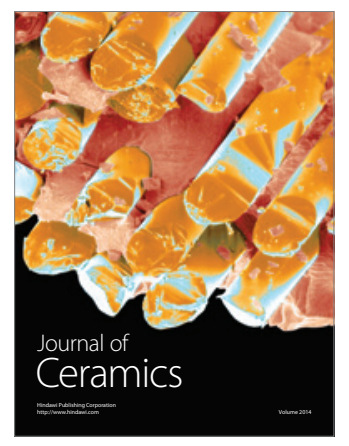

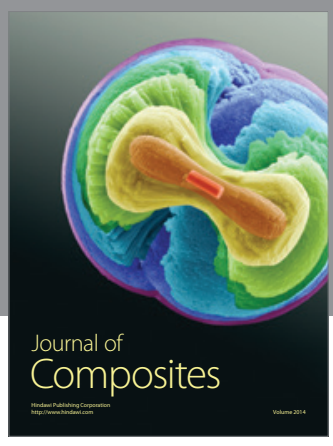
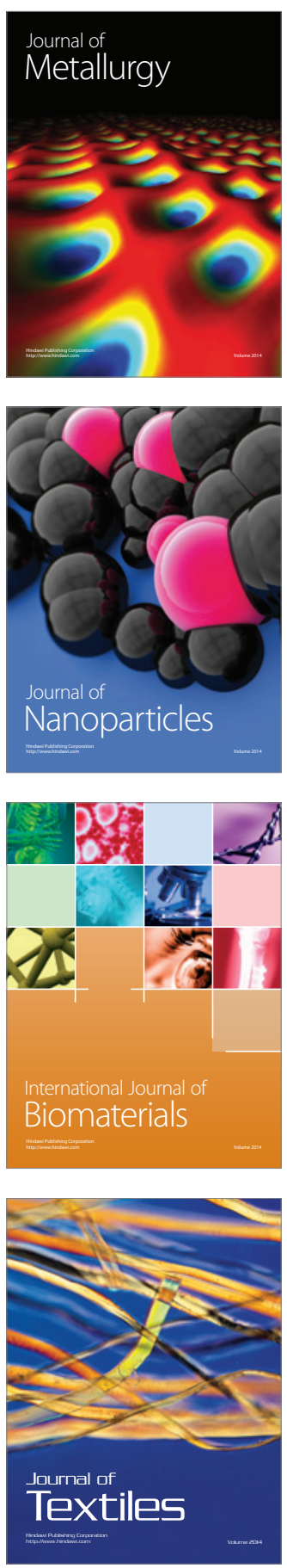